\title{
Effect of an Educational Program on Nurses' Knowledge and Practice Regarding Defibrillation and Cardioversion
}

\author{
Safaa Mahmoud Ahmed ${ }^{1}$, Zienab Abd El-Latif Muhammad², Hosam Hasan-Ali ${ }^{3}$, Mona Aly Muhammad ${ }^{4}$ \& \\ Mogeda Muhammad Mehany ${ }^{5}$. \\ 1. A professional Nurse in coronary care unit, Egypt. \\ 2. Professor of Medical, Surgical Nursing. Faculty of Nursing, Assiut University, Egypt. \\ 3. Professor in cardiovascular medicine Faculty of medicine, Assuite University, Egypt \\ 4. Assistant professor in Critical Care Nursing, Faculty of Nursing, Assiut University, Egypt. \\ 5. Assistant professor in Critical Care Nursing, Faculty of Nursing, Assiut University, Egypt.
}

\begin{abstract}
Electrical cardio version and defibrillation is a critical treatment for the survival of patients with cardiac arrest. Therefore, critical care nurses not only have to know how to monitor and operate the defibrillator, but also to provide interventions for preventing possible complications. The Aim of the present study was to evaluate the effect of educational program on knowledge and practice of nurses regarding defibrillation and cardio version. Method A convenient sample of 50 nurses working in coronary care unit and emergency care unit at Assiut University Heart Hospital. A structured interview questionnaire sheet and Nurses practice observational checklist tools were used to collect the data. Results the present study revealed that total mean scores of the nurses knowledge and practices were improved from a preprogram level of $(26.04 \pm 9.51)$ to knowledge and $(75.54 \pm 10.90)$ for practice to an immediate post of $(68.84 \pm 1.75)$ for knowledge and $(134.70 \pm 1.52)$ for practice. Conclusion Nurses' level of knowledge and practice in caring of patients was unsatisfactory before the program and significantly improved immediately post program and at follow up phase in all items. Recommendation: Orientation program should be utilized for newly graduated nurses'.
\end{abstract}

\section{Keyword: Nurse's Practices, Knowledge, Defibrillation, Cardioversion \& Life Threatening Dysrhythmia.}

\section{Introduction}

Defibrillators are life-saving and their early use improves survival of patients presenting with sudden cardiac arrest due to shockable rhythms if they are used safely and correctly. Of all health care professionals as critical care nurses' especially in coronary care unit are often faced with life and death situations and their ability to act quickly and efficiently is of paramount importance. Unless definitive action is taken, the patient will suffer irreversible brain injury. (Dulandas \& Brysiewicz, 2018)

The role of critical care nurse in life threatening dysrhythmias is the ability to detecting types of dysrhythmia during cardiac monitoring and rapidly assessment of patient for use of corrective action, obtaining 12- lead ECG to identify the type of threatening dysrhythmias, and delivering adequate oxygen to reduce heart workload. In cases such as atrial fibrillation, ventricular tachycardia, ventricular fibrillation and cardiac arrest, the nurse should perform rapid and safe defibrillation and other cardiac life support protocols to preserve oxygen supply to vital organs .( Khalil et al., 2018)

Despite the extension of training and the authorization of nurses to perform early defibrillation (advocated by the American Heart Association), such

practice has not been widely adopted in hospitals. Lack of: continuing educational programs or sessions about cardiovertion and defibrillation, supervision and continuous evaluation of nurses' practice. So the nurses are not able to diagnosing pulseless fibrillation and ventricular tachycardia; for this reason the nurses have inability to do the defibrillation shock in hospitals. (Abd El naeem et al., 2016)

Educational program for nurses regarding defibrillation and cardiovertion it is very important to meet the requirements of this complex role and cardiac devices to be able to meet the requirements and effects of patients' outcome or reduced of complication. So the critical care nurses are needed for continuous training to improve of nursing care and practices for critical ill patient. (Ruhwanya et al., 2018)

\section{Significance of the Study}

From researchers' clinical experience, it has been observed that critical care nurses are frequently the first responders and they witness nearly all the cardiac arrest in the unit. Also, according to patients' statistical record of coronary care unit, Assuite university heart hospital number of patients' admission during 2015 were (1144) per year. This high follow to coronary care unit at Assuite university heart hospital across Upper Egypt which 
admitted patients' with cardiac disease. So the trained Critical care nurses were able to help correctly diagnose life threatening dysrhythmia and early detection of cardiac arrest, and the need to defibrillate, and execute sequential steps to complete defibrillation. Successful defibrillation delivered by the nurses facilitated the smooth completion of the procedure and avoidance of complications. They should have knowledge, good practice and be skillful in dealing with this complication. So there is a need for a study to promote the nurses' knowledge and practice that could help them to contribute successful patients' care outcomes.

\section{Aim of the study}

The aim of the study is evaluate effect of an educational program on nurses' knowledge and practice regarding defibrillation and cardio version

\section{Research hypotheses}

To fulfill the aim of this study, the following research hypotheses were formulated:

$\mathrm{H}_{1}$. There will be a significant difference between post-test knowledge scores and the pre-test knowledge scores.

$\mathrm{H}_{2}$. There will be a significant difference between the post-test practices scores and the pre-test practices scores.

$\mathrm{H}_{3}$.A positive relation will exist between knowledge and practices score obtained by critical care nurses' receiving educational program.

\section{Subjects \& Method}

\section{Study design}

A quasi-experimental study design was used for conduction of this study.

\section{Study variables}

- The independent variable is the educational program.

- The dependent variable are critical care nurses' knowledge and skills.

\section{Setting}

The study was conducted in coronary care unit and emergency care unit at Assiut University Heart Hospital.

\section{Subjects}

A convenience sample of 50 nurses working in the above mentioned selected setting.

\section{Study tools}

Two tools were utilized for data collections: To assess the nurses, knowledge and practice regarding defibrillation and cardioversion. Each nurse was evaluated three time before, immediately (post- test) and 3 months after the program implementation and these were compare to pre - test level by using the same pre -test study tools (knowledge sheet and observational checklist ). They included
Tool one: Structured interview questionnaire sheet: This tool was developed by researcher after reviewing literatures to assess the knowledge level of critical care nurses regarding cardioversion and defibrillation. It was in Arabic language. This tool included two parts:

Part I: Socio-demographic data of study sample which included: age, sex, marital status, level of education, years of experience and attendance of previous training course.

Part II: Nurses' knowledge about anatomy and physiology of the heart, ECG interpretation, Cardioversion, Defibrillation. It consisted of 40 multiple choice questions. Scoring system: A total score of the questionnaire was 70 grades. All questions was measured and divided by the number of questions to obtain the mean knowledge of each nurse. Knowledge below $75 \%$ was considered unsatisfactory while those equal to or above $75 \%$ was considered satisfactory.

Tool II: Nurses practice observational checklist: This tool was adopted from (Fuzzed, 2016) for assessment of nurse's practices. Tool covered the steps. This tool covering different aspects of procedure for cardioversion and defibrillation composed of 24 checklists were included 50 steps.

Scoring system for nurses' practices: Every step was evaluated as follows; correctly done was scored (2), while incomplete answer was scored (1) and not done was scored (0). All questions were measured and divided by the number of questions to obtain the mean practice of each nurse. Below $75 \%$ was considered unsatisfactory while those equal to or above $75 \%$ was considered satisfactory.

\section{Method}

Preparatory phase and administrative design

- An official permission to conduct the study was obtained from the hospital responsible authorities after explanation of the nature and aim of the study.

- An approval was obtained from the local ethical Committee and the study was followed the common ethical principles in clinical research.

- The tools used in this study were developed by the researcher based on reviewing the relevant literature.

- Content validity: The tools were tested for content validity by jury of 5 experts in the field of critical care nursing and critical care medicine from Assiut University heart hospital, and the necessary modification were done.

- The reliability: Was tested for tool one (Knowledge assessment tool), and tool two. 
- (practice assessment tool) by using Cronbach,s coaefficient alpha $(\mathrm{r}=0.805,0.740)$

Pilot of study

- A pilot study was conducted on $10 \%$ nurses' to test the feasibility and applicability of the tools. After obtaining permission from the authorities of hospital management. After pilot study assessment has been done after making necessary corrections in the questionnaire.

\section{Ethical considerations}

An officially letter from the faculty of nursing was send to accountable authorities of the hospital and approval was attained to conduct this study after explanation of the aim of the study. Formal consent was taken from the nurses to participate in the study. The researchers firstly introduced themselves to all potential subjects, then explaining the purpose of the study and they were assured that all data collected would be very confidential and only will be used for the study' aim. The researchers stressed that contribution in the study are entirely volunteer and anonymity of the nurse was sure through coding data. Subjects were also knowledgeable that rejection to participate in the study would not disturb their care.

The study was implemented through 4 phases

Assessment: Upon finalization of the tools and getting official permission the researcher stated to assess nurses knowledge by using structured interview questionnaire, this was followed by observing their practice in different shift using the observational checklist.

Planning: Based on analysis of the collected data and using pertinent literature the researcher developed an educational program in form of hand out booklet in Arabic language. The objective of the program was to improve nurses' knowledge and practice regarding emergency care of patients undergoing Defibrillation and cardiovertion. The program was developed to cover all necessary knowledge and practice needed for proper practice.

Implementation: The program was implemented for the ten subgroups of nurses. All groups were exposed to four session's in-addition to the preliminary one. Preliminary session: In this session the researcher met the participants and explained the objectives, contents, and methods of evaluation of program. Since the researcher work in the same place, more than one session could be given each day.

Session I included: Anatomy and physiology of the heart. For duration of 30 minutes.

Session II included: Identify the ECG Basics, how calculate heart rate. For duration of 30 minutes.

Session III included: life threatening arrhythmias, Definition, causes, nurses role. For duration of 1 hour.
Session IV included: Defibrillation and cardiovertion, definition, types, indication, precaution, complication, nurses role, definition of sudden cardiac arrest (SCA), signs and symptoms. For duration of 2 hour.

- Each session usually started by a summary of what has been taught during the previous sessions and the objectives of the new topics.

- Group discussion was encouraged with continuous feedback to ensure understanding and achievement of the specific objective of the program.

- An open channel of communication was established between the researcher and nurses' to answer any question and reinforce the gained information and correct actions.

- In the last session the researcher summarized and emphasized the important points.

- Each nurse obtained a copy of the teaching protocol booklet that included all the training contents.

- Learning environment: The program was conducted in the head nurses' office in coronary care unit at hospital.

- Teaching methods: Lectures and discussion, demonstration and re-demonstration by using audiovisual aids.

- Teaching facilities: Power point presentation, booklet which developed in Arabic language by the researcher based on reviewing the related literature, videos about electrical conduction system of the heart, cardioversion procedure, and cardiopulmonary resuscitation basic life support (BLS).

Assess nurses' practices

The researcher observes the nurses' practices using observational checklist tool twice before, immediately (post- test) and 3 months after the program implementation.

\section{Limitation of the study}

It was very difficult to gather all nurses at the same time, so, the program was applied on 6 successive months.

\section{Statistical analysis}

Date entry and data analysis were done using SPSS version 19 (Statistical Package for Social Science). Data were presented as number, percentage, mean, standard deviation. Chi-square test and Fisher Exact test were used to compare qualitative variables. Pearson correlation was done to measure correlation between quantitative variables. P-value considered statistically significant when $\mathrm{P}<0.05$. 


\section{Result}

Table (1): Frequency distribution of socio demographic data of the studied nurses $(\mathrm{No}=50)$.

\begin{tabular}{|c|c|c|}
\hline Variables & No. (50) & $\%$ \\
\hline \multicolumn{3}{|l|}{ Age: (years) } \\
\hline$<25$ & 27 & 54.0 \\
\hline$\geq 25$ & 23 & 46.0 \\
\hline Mean \pm SD (Range) & \multicolumn{2}{|c|}{$24.94 \pm 3.62(21.0-34.0)$} \\
\hline \multicolumn{3}{|l|}{ Gender: } \\
\hline Male & 2 & 4.0 \\
\hline \multicolumn{3}{|l|}{ Marital status: } \\
\hline Single & 17 & 34.0 \\
\hline Married & 33 & 66.0 \\
\hline \multicolumn{3}{|l|}{ Qualifications: } \\
\hline Secondary school of nursing & 11 & 22.0 \\
\hline Nursing technical institute & 31 & 62.0 \\
\hline Bachelor of Nursing & 8 & 16.0 \\
\hline \multicolumn{3}{|l|}{ Years of experience: } \\
\hline$<5$ & 35 & 70.0 \\
\hline$\geq 5$ & 15 & 30.0 \\
\hline Mean \pm SD (Range) & \multicolumn{2}{|c|}{$5.36 \pm 4.94(1.0-17.0)$} \\
\hline Training about cardioversion and defibrillation: & & \\
\hline Yes & 7 & 14.0 \\
\hline No & 43 & 86.0 \\
\hline
\end{tabular}

Table (2): Mean score distribution of studied nurses' Knowledge and practices before, after and follow up implementation of educational program.

\begin{tabular}{|l|c|c|c|c|c|c|}
\hline \multirow{2}{*}{$\begin{array}{l}\text { Knowledge and } \\
\text { practices score }\end{array}$} & \multirow{2}{*}{$\begin{array}{c}\text { Total } \\
\text { score }\end{array}$} & $\begin{array}{c}\text { Before program } \\
(\mathbf{N}=\mathbf{5 0})\end{array}$ & $\begin{array}{c}\text { After program } \\
(\mathbf{N}=\mathbf{5 0})\end{array}$ & $\begin{array}{c}\text { Follow-up } \\
(\mathbf{N}=\mathbf{5 0})\end{array}$ & $\begin{array}{c}\text { P- } \\
\text { value }^{1}\end{array}$ & \multirow{2}{*}{$\begin{array}{c}\text { P- } \\
\text { value }^{2}\end{array}$} \\
\hline $\begin{array}{l}\text { Total score } \\
\text { Knowledge }\end{array}$ & $\mathbf{7 0}$ & $26.04 \pm 9.51$ & Mean \pm SD & Mean \pm SD & & \\
\hline $\begin{array}{l}\text { Total score } \\
\text { practices }\end{array}$ & $\mathbf{1 3 6}$ & $75.54 \pm 10.90$ & $134.70 \pm 1.52$ & $131.98 \pm 4.17$ & $0.000^{*}$ & $0.000^{*}$ \\
\hline
\end{tabular}

* Statistical significant difference $(P<0.05), P$-value ${ }^{I}$ between pre \& post, $P$-value ${ }^{2}$ between pre \& follow up, Independent samples t-test

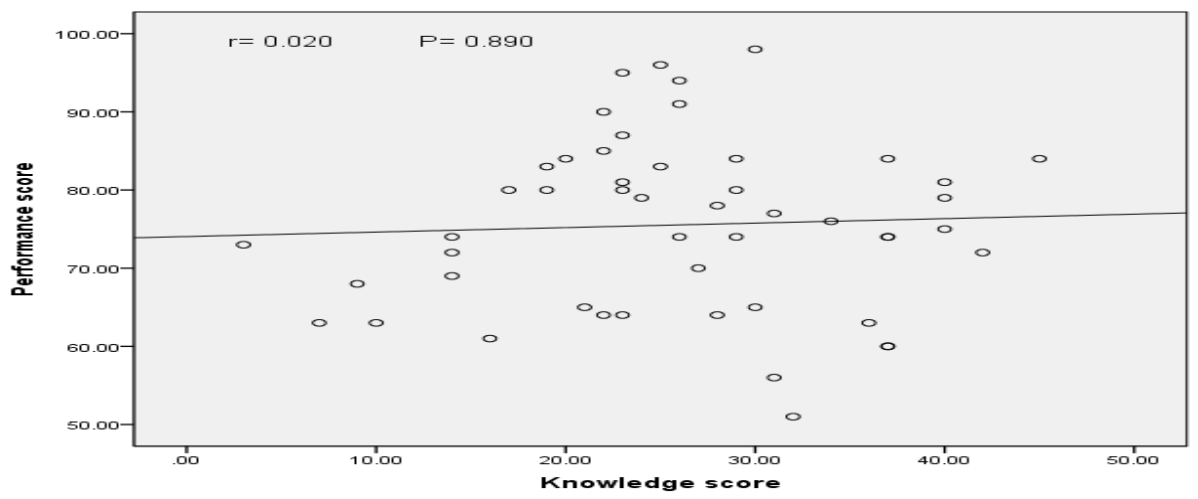

Figure (1): Correlation between total nurses' knowledge score and total of nurses' practices score before implementation of the educational program.

Statistical significant difference $(P<0.05) \quad$ Pearson $t$-test 


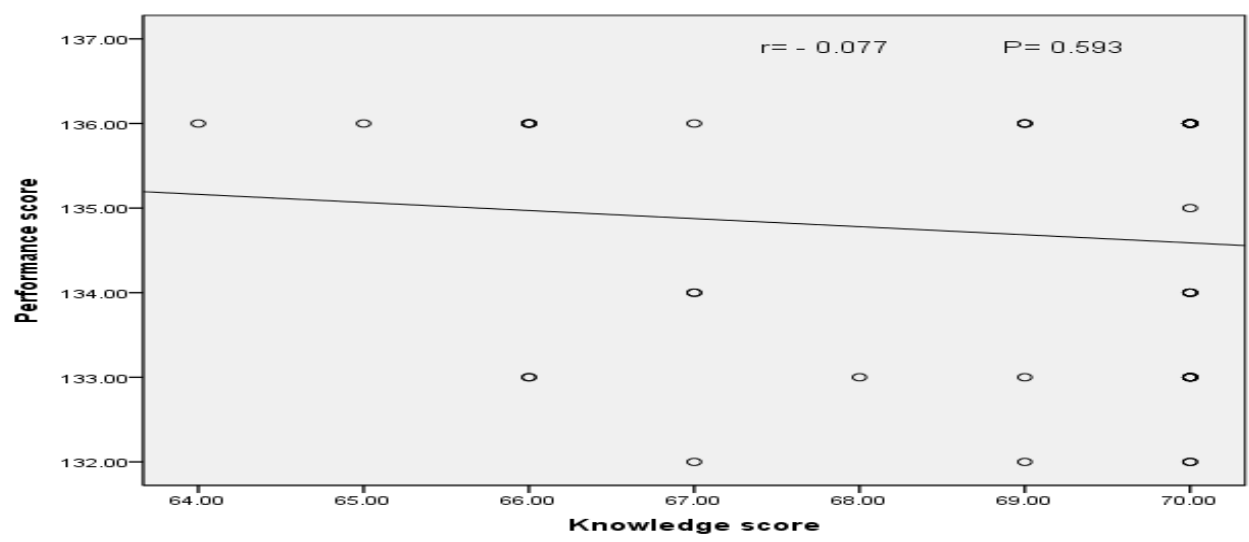

Figure (2): Correlation between total nurses' knowledge score and total of nurses' practices score after implementation of the educational program.

Statistical significant difference $(P<0.05), \quad$ Pearson t-test

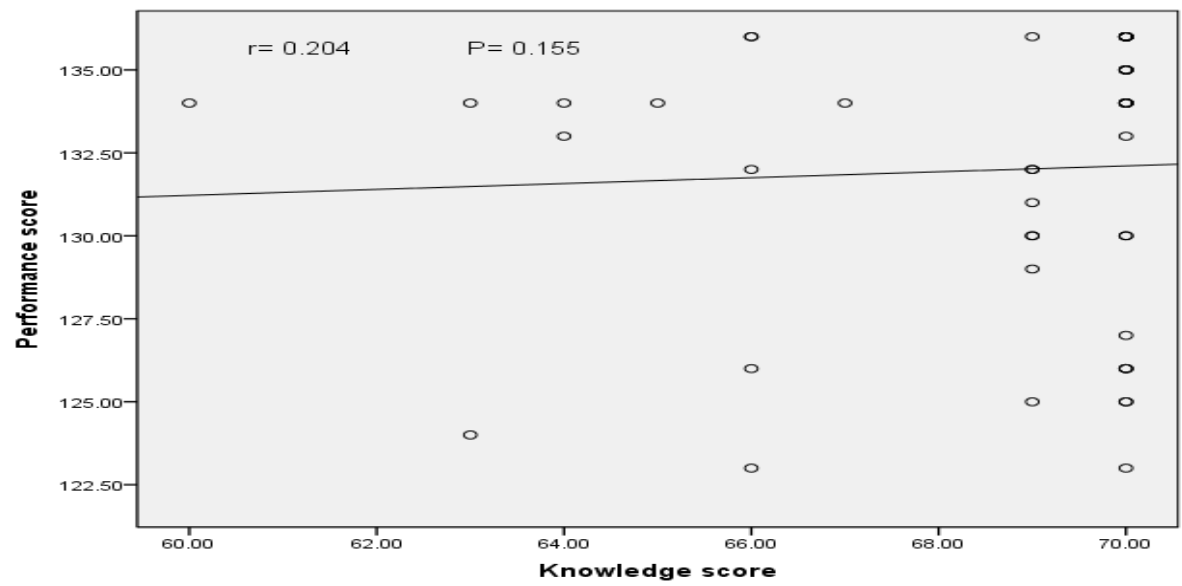

Figure (3): Correlation between total nurses' knowledge score and total of nurses' practices score before and follow up implementation of the educational program.

Statistical significant difference $(P<0.05), \quad$ Pearson $t$-test.

Table (1): This table shows socio-demographic characteristics of the nurses, it was founded that , more than half of them (54.0\%) their age is less than 25 years with mean $\pm \mathrm{SD}$ ( $24.94 \pm 3.62)$. While the majority of them were females $(96.0 \%)$. More than half of the studied nurse had were married $(66.0 \%)$ .More than half of the studied nurse had were technical health institute in nursing $(62 \%),(22 \%)$ of the nurses held secondary school of nursing , $16 \%$ Bachelor of Nursing. Moreover most of the nurses had not received any previous training, the table reveals that $(86 \%)$. Concerning their experiences it was found that nearly three quarter $(70 \%)$ were less than five years of experiences with mean \pm SD $(5.36$ $\pm 4.94)$.

Table (2): Shows total mean scores of nurses' knowledge and practice concerning cardio version and defibrillation before, after and follow up educational program. It was apparent from these table of the total mean scores of Knowledge immediately after program was $(68.84 \pm 1.75)$ higher than the pre program with mean score $(26.04 \pm 9.51)$ while mean scores of the nurses knowledge at 3 months follow up was $(68.56 \pm 2.43)$. Also, total mean scores practice in the immediate after program ( $134.70 \pm$ 1.52) was higher than the pre program with mean score $(75.54 \pm 10.90)$, while mean scores of the nurses knowledge at 3 months follow up (131.98 \pm 4.17) . It was found from these table there were a statistically significant improvement were noticed in nurses Knowledge and practices between before educational program and after educational program and between before and follow up . P $<0.001$

Fig (1, 2 and 3): Show that there was no significant correlation between nurses' knowledge score and total of nurses' practices score before, after and follow up implementation of the educational program regarding cardio version and defibrillation. 


\section{Discussion}

Defibrillation and cardioversion have been traditionally regarded as skills performed exclusively by medical staff, it has become routine practice for nurses to defibrillate and initiate resuscitative measures during cardiac arrest. (Bradley et al., 2017) It can source of various health problems that have to be recognized and treated following the test. Taking into consideration probable dangerous complications so that the nurse plays a critical role in coronary care unit in the provision great quality nursing care and increase patient safety to their patients. Creature knowledgeable and existing on evidence based practice is the crucial to being an efficient and effective nurse. So nurses should understand clearly information and optimal performance about patient safety to improve quality care.

In the present study, the majority of the nurses were in the age group young adults. More than half of them had less than 5 years of experience. This result may be attributed young adulthood can be considered as a period of stabilization in which the individual makes career decision and becomes more adaptable and responsive to challenges. In this respect, (Maville \& Huerta, 2012) who told that young adult behavior during provision of medical care, they become more aware of the impact of medical care on the patients; sensitive to their fears; honestly explain medical procedures and expected outcomes. Also, this finding agrees with (El Mola \& Mohamed, 2018) who found that more than three-quarters of the studied sample have less than 5 years of experience. The result of study agrees with Rohith (2012). Moreover majority of the nurses' in the current study had not previous training in coronary care unit. This finding agrees with Laxman (2014) who reported that most of the nurses did not receive any special education related to the use of defibrillators before program. In the same line Mohamed (2010) who told that all of studied nurses' didn't attend any training program

The study findings revealed that, more than half of the nurses' were from nursing technical Institute. Finding of the present study is in agreement with El Mola \& Mohamed (2018) who reported that more than half of them were graduated from the Technical School of Nursing and the Technical Institute of nursing. However, this finding is contradicted by Kader \& Ali (2012) who told that critical care nurses' knowledge and practice of fever management at a university hospital and revealed that more than two thirds of the studied sample had bachelor degree. These differences "from the researcher's point of view" may be related to different types of nursing recruitment and variation of patient acuity level in the selected

ICUs.
In the present the majority of the studied nurses were females attribute. This result was strongly supported by Laxman \& Mohamed (2014) who stated that more than half of the studied sample was females. And agreed with Hamato \& Fadlallah (2015) who found that most participants were females. Also agreed with El Mola \& Mohamed (2018) who found that two-thirds of the studied samples were women, and in this respect Rashdan (2007) who conducted a study about implications for advancement of Egyptian nursing, and found that nursing in Egypt is primarily females occupation and very few men are admitted to nursing programs in the university sector, Also Dis agreement with Hassan \& Aburaghif (2016) who stated that most participant of the study sample were males.

The present study revealed the total mean score of nurses' knowledge before implementation of the educational program generally was poor. This may be due to they had not receive previous training program about cardio version and defibrillation. The reasons for lack of knowledge pertinent to cardio version and defibrillation "from the researcher's point of view" may be related to lack of: continuing educational programs or sessions about this therapeutic intervention, supervision, continuous evaluation of nurses' practice, and cooperation between multidisciplinary health care team members. Another cause for lack of knowledge nurses' exhaustion due lack of Arabic nursing text book and they overloaded by their work which may hinder their ability to read and update their knowledge, no pre-employment orientation program, insufficient number of nurses, inadequate salary. This result was in line with Gupta \& Dias (2014) who stated that the results of pre test of the study reveal that there is low level of knowledge and practice scores about the defibrillation technique.

In the same line (Sara et al., 2016) who stated that to identify critical nurses' perception of arrhythmia knowledge that there is a deficit in nurses' ability to recognize and identify specific arrhythmia pre educational program. Also, this present finding was in line with Sheilini \& Devi (2014) who found that lack of nurses' knowledge about ECG and its interpretation was documented by the results of the pre test. Also, Hassan \& Hassan ( 2013) who reported that the majority of nurses' had unsatisfactory knowledge and practice before implementation of the educational program and after applying the education program there were improvement .This is in line with Thomas (2013) who revealed that a deficit in nurses' ability to recognize and identify specific arrhythmias including heart block, aberrant conduction, and tachyarrhythmia. 
Moreover, after implementation of the educational program the improvement was shown in nurses' knowledge mean score regarding all aspects of the program, similar improvements was also revealed in the nurses' practice. Also the follow up mean score (after 3 months) had shown some decline but they were still significantly higher compared to pre program level. This decline in the total mean scores of nurses' knowledge and practice in the follow phase is normal and expected finding. This result was in agreement with (Ibrahim et al., 2017) \& Refaey (2012) who stated that the mean knowledge scores of nurses are increased immediately after implementation of the program with asignificant statistical difference.

As regards nurses' practices related to defibrillation, the present study showed that there was improvement after implementation of standards compared with before implementation. This result is supported by (Abedellneem et al., 2016) \& Taha (2017), who reported that the nurses had adequate practice scores after program implementation, with a highly statistically significant difference.

Also, (Abd-Elkareem et al., 2012) who mentioned that the nurses' performance in CPR was not satisfactory before using the nursing procedures manual but was satisfactory afterwards. Regarding Correlation between total knowledge and practice score, the present study found that there was no statistical significance difference between total nurse's level of knowledge and their practice regarding cardio version and defibrillation.. In the same line, El Shafaey \& Abdel Aziz (2018) who found in their study about "Nurses Knowledge and their practice regarding postoperative cataract patients no statistical significance difference relation between total nurse's level of knowledge and their practice. This result may be due to that absence of inservice training program required for update nurses' knowledge, lack of guidance and supervision from the head nurse, and absence of booklet being able to affect their practice. On the country, this result disagrees with Shehab et al (2018) who found significant relation between total nurse's level of knowledge and their practice.

\section{Conclusion}

After educational program, the defibrillation and cardiovertion were significantly for gain in knowledge and practice scores.

Recommendation: In the light of the study findings, the following recommendations were suggested

- Orientation program should be utilized for newly graduated nurses in critical care units to improve their knowledge and practice.
- Nurses in critical care units should updating their knowledge and practice through frequent attending seminars, and conferences based on their needed assessment.

- Continous educational program for theses nurses concerning any emergency situation in the critical care units.

\section{Reference}

1. Abd El naeem, M., Mohamed, N., Mohammed, M., \& Abd El-Aziz, M., (2016): Effect of Teaching Program on Knowledge and Skills Regarding Automatic External Defibrillation among Nurses Working In Emergency Unit, ,Volume 5, Issue 1, PP. 08-1 .

2. Abd-Elkareem H., Adam S., \& Hassan R., (2012): Effect of nursing procedures manual on nursing performance in the intensive care unit. New Egypt J Med ,46:231-232.

3. Bradley P., (2018): Patient education: Cardio version, all topics are updated as new evidence becomes available and our peer review process is complete. Literature review current through: Jun 2018. | This topic last updated: Dec 04.

4. Dulandas, R., \& Brysiewicz, P., (2018): A description of the self-perceived educational needs of emergency nurses in Durban, KwaZulu-Natal, South Africa. African Journal of Emergency Medicine, 8(3), PP.84-88.

5. El Mola, M., \& Mohamed, S., (2018): Staff nurses expected role versus actual role at South Valley University. Egyptian Nursing Journal, 15(1), P.62.

6. El Shafaey \& Abdel Aziz, B., (2018): Effect of Implementing Teaching Program on Knowledge and Practice of Nurses and Clinical Outcomes of Patients Post Cataract Surgery, Journal of Nursing a nd Health Science, 7 (3) , PP. 60-70

7. Fuzzed, K., (2016): Education Curriculum for Advanced Life Support (ALS). Internal document: Bendigo Health.

8. Gupta, V., \& Dias, R., (2014): Effectiveness of Simulated Demonstration regarding Defibrillation Technique on Knowledge and Practices among Nurses. International Journal of Science, 3 (6), PP. 720-727

9. Hamato \& Fadlallah, M., (2015): Nurses' Knowledge and Practice Regarding Nursing Care for Myocardial Infarction Patients in the first 24 hours in Governmental Hospitals in Khartoum state, The National Ribat University ,College of Post Graduate studies \& Scientific Research .P.64 
10. Hassan, N., \& Aburaghif, L., (2017): Effectiveness of an Educational Program on Nurse's knowledge concerning Complications of Cardiac Catheterization among Children at AlNassirhya Heart Center. Kufa Journal for Nursing Sciences, 6(3), PP.161-170

11. Hassan, S., \& Hassan, H., (2013): Effectiveness of Nursing Education Program on Nurses Practices Toward Arrhythmia in Kirkuk's Teaching Hospitals. kufa Journal for Nursing sciences, 3(1), PP.220-230.

12. Ibrahim, R., Abd-Allah, K., Arafa, O., \& Mohammed, S., (2017): Effect of nursing care standards on nurses' performance in caring for patients with cardiac arrhythmias. Egyptian Nursing Journal, 14(3), P.251.

13. Kader Mohamed, L., \& Ali, N., (2012) Critical Care Nurses' Knowledge and Practice of Fever Management at a University Hospital. Journal of American Science, 8(12), PP.1545-1553

14. Khalil, N., Rahman, H., \& yaser Hamouda, E., (2018): Critical care nurses' knowledge and practice regarding life-threatening ventricular dysrhythmias. Clinical Practice (Therapy), 15(4), PP.747-753.

15. Laxman, M., (2014): Study of knowledge of staff nurses regarding use of defibrillators in intensive care units. Vol.-4, Issue- 1, PP. 298306.

16. Maville, J., \& Huerta, C (2012). Health Promotion in Nursing: Cengage Learning, (3rd Ed) ,PP.223.

17. Mohammed M., (2010): knowledge, practices and attitude of nurse's toward universal precaution in outpatient clinics Assiut University Hospital.

18. Rashdan, T., (2007): Implications for advancement of Egyptian nursing: input equals output. In White paper for Fulbright academy workshop in Doha, March, PP. 23-25.

19. Refaey, A., (2012): Impact of Adesigned Teaching Protocol about Advanced Cardiac Life Support (ACLS) On Critical Care Nurse's Knowledge and Practices at Benha University Hospital, Cairo, Egypt. J Am Sci, 8(12), pp.83850.

20. Rohith RAO A., (2012): "A study to assess the effectiveness of structured teaching programme on knowledge of defibrilation among the staff nurses in selected hospitals at tumkur" nursing sri siddhartha college of nursing, agalakote, tumkur, rajiv gandhi university of health sciences .PP. 9-14

21. Ruhwanya, D., Tarimo, E., \& Ndile, M., (2018) Life threatening arrhythmias: Knowledge and skills among nurses working in critical care settings at Muhimbili National Hospital, Dar es Salaam, Tanzania. Tanzania Journal of Health Research, 20(2).

22. Sara, H., Zedaan, H., Ahmed, R., Owaid, H., \& Mousa, A., (2016): Nurses' Knowledge Concerning Early Interventions for Patients with Ventricular Tachycardia at Baghdad Teaching Hospitals. kufa Journal for Nursing sciences, 6(2), PP.8-15.

23. Shehab, M., Ibrahim, N., \& Abd-Elkader, H., (2018): Impact of an Educational Program on Nurses' Knowledge and Practice Regarding Care of Traumatic Brain Injury Patients at Intensive Care Unit at Suez Canal University Hospital. International Journal of Caring Sciences, 11(2), P.1104.

24. Sheilini, M., \& Devi, E., (2014): Effectiveness of educational intervention on ECG monitoring and interpretation among nursing students. Journal of Dental and Medical Sciences, 13 (12), PP.01-05.

25. Statistical Record (2015): Patients' in coronary care unit at Assuite university heart hospital number of patients' admission during, (2015) were 1144 per year.

26. Taha, A., (2017): Impact of a Designed Teaching Protocol about Nursing Management of Coronary Artery Bypass Grafting on Nurse's Knowledge, Practices and Patient's Outcome, Benha University, Egypt , Journal of Nursing and Health Science, Volume 6, Issue 4 Ver. III, PP. 13-28 .

27. Thomas, M., (2013): A study to evaluate the effectiveness of video assisted teaching programme on knowledge and practice regarding cardioversion and defibrillation among staff nurses working in cardiac unit in selected hospitals of Bagalkot (Doctoral dissertation). 